\title{
Percepção dos usuários de uma Estratégia de Saúde da Família sobre o Acolhimento
}

\author{
Perception of the users of a Family Health Strategy on the host \\ Percepción de los usuarios de una Estrategia de Salud Familiar en el huésped \\ Michele Aparecida Silva Maciel ${ }^{1 *}$, Patrícia Costa dos Santos da Silva ${ }^{1}$, Lívia Ferreira Oliveira.
}

\begin{abstract}
RESUMO
Objetivo: Conhecer o acolhimento na percepção dos usuários de uma estratégia de saúde da família. Métodos: O estudo é de natureza qualitativa, descritiva e exploratória. A coleta dos dados ocorreu por meio de entrevista estruturada. Como critérios de inclusão, os usuários necessitavam ser maiores de 18 anos, com registro na UBSF. A coleta de dados foi efetivada por meio de um roteiro prévio, desenvolvido pelos pesquisadores, com a finalidade de identificar a condição socioeconômica dos usuários. Resultados: Os entrevistados possuem em média uma renda mensal de um a dois salários mínimos, a maioria era do sexo feminino entre a faixa etária de 20 a 69 anos. Quanto à escolaridade constatamos que o maior percentual dos participantes declararam ensino médio completo. A análise temática possibilitou a construção de duas unidades: 0 atendimento com qualidade $e o$ atendimento quanto à quantidade e ao tempo na Atenção Primária à Saúde. Conclusão: $O$ usuário busca um ambiente de saúde resolutivo que organiza uma rica ação de retroalimentação, ou seja, uma via de mão dupla, na qual usuário e trabalhadores participam do processo de saúde.
\end{abstract}

Palavras-Chave: Acolhimento, Sistema Único de Saúde, Percepção.

\begin{abstract}
Objective: To know the reception in the perception of users of a family health strategy. Methods: The study is qualitative, descriptive and exploratory. Data collection occurred through structured interviews. As inclusion criteria, users needed to be over 18 years old, registered with UBSF. Data collection was performed through a previous script, developed by the researchers, in order to identify the socioeconomic status of users. Results: Respondents have, on average, a monthly income of one to two minimum wages, most of them female between the ages of 20 and 69 years. Regarding education, we found that the highest percentage of participants declared complete high school. The thematic analysis enabled the construction of two units: quality care and care regarding quantity and time in Primary Health Care. Conclusion: The user seeks a resolute health environment that organizes a rich feedback action, that is, a two-way street in which users and workers participate in the health process.
\end{abstract}

Keywords: Reception, Health Unic System, Perception.

\section{RESUMEN}

Objetivo: Conocer la acogida en la percepción de los usuarios de una estrategia de salud familiar. Métodos: El estudio es cualitativo, descriptivo y exploratorio. La recolección de datos se realizó a través de entrevistas estructuradas. Como criterios de inclusión, los usuarios debían ser mayores de 18 años, con inscripción en la UBSF. La recolección de datos se realizó a través de un guión anterior, desarrollado por los investigadores,

${ }^{1}$ Universidade Federal de Uberlândia. Uberlândia, MG - Brasil. *E-mail: michele_unitri@hotmail.com 
para identificar el estado socioeconómico de los usuarios. Resultados: los encuestados tienen, en promedio, un ingreso mensual de uno a dos salarios mínimos, la mayoría mujeres de 20 a 69 años. Con respecto a la educación, encontramos que el porcentaje más alto de participantes se declaró en la escuela secundaria completa. El análisis temático permitió construir dos unidades: atención de calidad y asistencia de cantidad y tiempo en atención primaria de salud. Conclusión: el usuario busca un entorno de salud resuelto que organiza una acción de retroalimentación enriquecida, es decir, Una calle de doble sentido, en la que usuarios y trabajadores participan en el proceso de salud.

Palabras clave: Recepción, Sistema único de Salud, La percepción.

\section{INTRODUÇÃO}

A partir da promulgação das leis ํo 8.080 e ํำ 8.142, de 1990, nasceu o Sistema Único de Saúde (SUS), o qual representa uma grande conquista nacional que, em meio aos desafios e entraves ocorridos ao longo de sua construção, permitiu uma nítida evolução e fortalecimento da Atenção Primária à Saúde (APS) no Brasil, o que, por sua vez, contribui sobremaneira para a implantação de seus princípios e de suas diretrizes (MOROSINI MV et al.,2018). Além disso, a APS é considerada como a porta de entrada desse novo modelo de atenção, sendo que as evidências científicas a apontam como uma estratégia de reorientação do serviço de saúde (LOPES AS et al., 2015).

Por outro lado, o Brasil possui ainda um longo caminho a ser percorrido, antes que seja atingido o alvo de se obter um sistema que se traduza na prática com a aplicação de uma clínica ampliada, com a participação de diferentes saberes, trabalhadores e áreas da política pública, de forma a transcender a lógica biologicista e enfrentar os determinantes sociais do processo saúde-doença (BACKES DS et al., 2014; MOROSINI MV, 2018).

Refletir sobre a APS como requisito primordial para a qualidade da assistência de saúde é de suma importância e, para que os avanços sejam alcançados, é crucial romper o paradigma de um sistema fragmentado. $\mathrm{O}$ acesso ao serviço de saúde ocorre muitas vezes por meio do nível secundário ou do terciário, em virtude de um desconhecimento por parte tanto dos usuários como dos profissionais de saúde acerca dos princípios e das diretrizes do SUS, além de conceberem o sistema de saúde vigente como dicotômico, pontual e linear em suas formas de atuação (BACKES DS et al., 2014).

Diante desse cenário, floresce a Política Nacional de Humanização (PNH) como proposta para efetivar os princípios do SUS no cotidiano da atenção à saúde e dar voz aos usuários, aos gestores e aos trabalhadores com a finalidade de melhoria do processo e da integração dos mesmos. Além disso, a prática do acolhimento por meio da escuta e do diálogo permeia o trabalho das equipes, conduzindo a um sentimento de compromisso e de vínculo assinalados pela intersetorialidade e pela interdisciplinaridade (SANTOS DDS et al., 2018).

Nesse sentido, o acolhimento emerge como uma ferramenta eficaz da $\mathrm{PNH}$, como diretriz e instrumento de reorganização do sistema, podendo acontecer a qualquer momento e local, por meio da escuta ativa dos usuários por todos os profissionais de saúde, estabelecendo-se vínculo e trazendo resolubilidade para os problemas levados para a Unidade Básica de Saúde da Família (UBSF). Dessa forma, o ator social entende seu papel e se torna consciente e participativo, dentro dessa política (BRASIL, 2004).

Compreende-se que o acolhimento é uma etapa do processo de trabalho, podendo ser realizado em um espaço físico determinado e por meio de uma equipe designada para atender à demanda da população adscrita (FERREIRA BR et al., 2018). Desse modo, o acolhimento passa a ser pensado como uma "relação de ajuda", em que os usuários são aqueles que procuram ajuda, sendo simultaneamente "objetos e agentes da ação", pois opinam e tomam decisões (BRASIL, 2013).

Assim, torna-se crucial que o usuário compreenda os princípios e as diretrizes que regem esse sistema, bem como que conheça o acolhimento em suas diferentes nuances, possibilitando que esse usuário seja de 
fato um protagonista nesse processo e busque de forma ativa seus direitos e deveres. Frente a essa temática, o presente estudo teve por objetivo conhecer a percepção dos usuários de uma estratégia saúde da família sobre o acolhimento (BRASIL, 2017).

\section{MÉTODOS}

Este estudo é de natureza qualitativa, descritiva e exploratória, a coleta de dados ocorreu por meio de entrevista estruturada iniciada em julho de 2018 e concluída em abril de 2019, o presente estudo integra um estudo maior intitulado Acolhimento e Resolubilidade das Situações Vivenciadas Pela Estratégia de Saúde da Família, executado junto ao grupo de pesquisadores do curso de graduação em Enfermagem da Universidade Federal de Uberlândia. As informações tiveram como fonte os usuários de uma Unidade Básica de Saúde da Família (UBSF), alocada no município de Uberlândia, do estado de Minas Gerais.

Para os critérios de inclusão, os usuários necessitavam ser maiores de 18 anos, com registro na UBSF, ter passado por atendimento nos últimos dois meses mediante a data da coleta e aceitar a realização da pesquisa. Os entrevistados foram orientados a respeito da mesma e dos seus objetivos, após as instruções assinaram o Termo de Consentimento Livre e Esclarecimento. Além disso foram explicadas questões sobre o sigilo das informações adquiridas e da possibilidade de futuras publicações científicas. De modo a preservar o anonimato dos participantes foi escolhido a numeração arábica

A coleta de dados foi efetivada por meio de um roteiro prévio, desenvolvido pelos pesquisadores, com a finalidade de identificar condições socioeconômicas dos usuários do serviço. Somado a isto, realizou-se uma entrevista estruturada que avaliou o conhecimento empírico do acolhimento por parte dos atores sociais. Para recurso documental, utilizou-se gravações com um aparelho celular. A pesquisa contou com 29 participantes, que responderam a entrevista em um tempo médio de 20 minutos, sendo efetuada por um dos elaboradores da pesquisa. As informações coletadas foram transcritas na sua totalidade, seguindo os critérios propostos por Minayo (2014), efetuando a organização e análise do material coletado, na qual ocorreu a categorização e escolha dos eixos temáticos, posteriormente, interpretação e ordenação dos dados.

Deste modo o estudo passou por análise e aprovação número 1.881.199. do comitê de ética e pesquisa.

\section{RESULTADOS E DISCUSSÕES}

Os entrevistados possuem em média uma renda mensal de um a dois salários mínimos, a maioria era do sexo feminino entre a faixa etária de 20 a 39 anos (Figura 1).

Figura 1 - Caracterização sócio demográfico dos usuários.

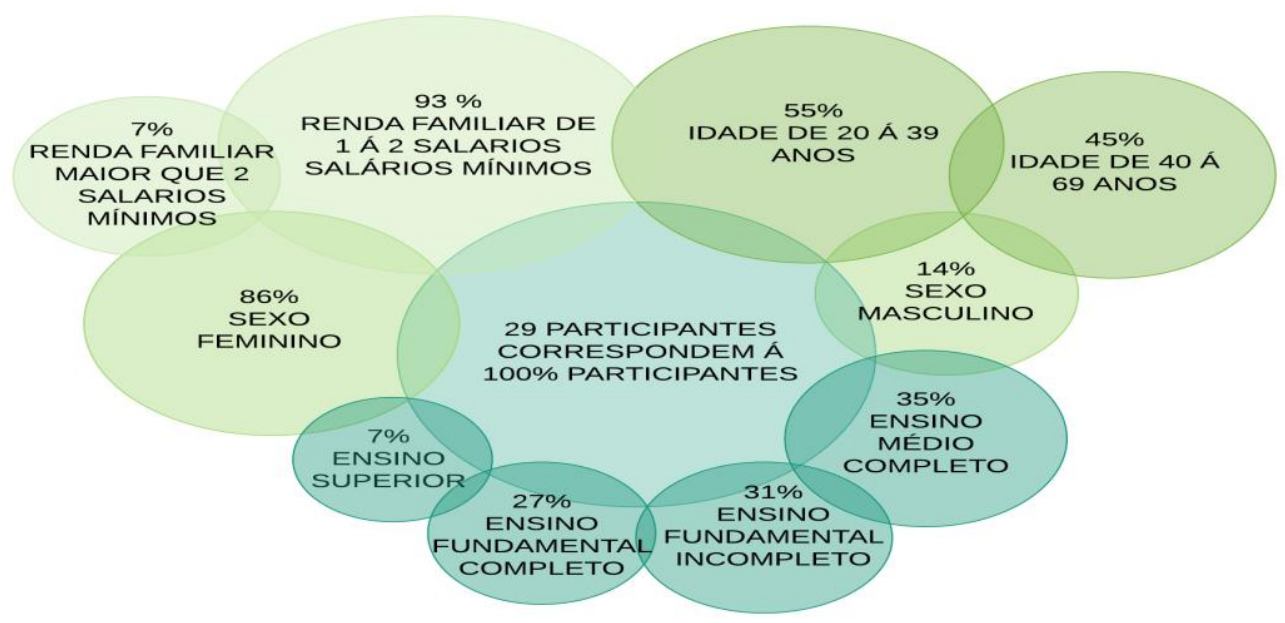

Fonte: Maciel MAS, Silva PCS, Oliveira LF, 2019. 
Quanto à escolaridade constatamos que o maior percentual dos participantes declararam ensino médio completo seguido pelo ensino fundamental incompleto e completo e por fim ensino superior como demonstrado na Figura 1.

A análise dos dados possibilitou a construção de duas unidades temáticas que serão apresentadas a seguir.

\section{$O$ atendimento com qualidade}

O acolhimento é percebido pelos entrevistados como um atendimento, em que o usuário busca o serviço e consegue ser recebido sem dificuldade, ou seja, a forma como ele é recepcionado no serviço, conforme salientam as falas:

Acolhimento para mim é o paciente chegar na unidade e ser atendido. Isso para mim é acolhimento, ser atendido sem pretexto, sem dificuldade [...] é o atendimento inicial, é aquele que vem antes da triagem, antes da consulta médica, vamos dizer atenção primária dentro do sistema de saúde [...] (21).

O bem-estar da pessoa você se sentir bem, bem recepcionada bem atendida (18).

Pra mim, é ser bem atendida, o médico me tratar bem, sempre estar a minha disposição para quando eu precisar (25).

É como a gente é recebido né tratado, acolher tentar tirar nossas dúvidas tentar alcançar o objetivo de ajudar para mim é isso (26).

Ainda pode-se identificar na percepção dos usuários quanto ao significado do acolhimento, que o consideram como uma via de mão dupla (Figura 2), caracterizada pelas falas de ser bem recebido em que tanto o usuário quanto o trabalhador devem caminhar juntos e de mãos dadas para o acolhimento. É um meio de emancipar o ator social de forma que ele possa participar da elaboração do plano de cuidados e ações que visem à prevenção, à promoção e a reabilitações das condições de saúde, efetivando-se o conceito de integralidade do SUS (BRASIL, 2007).

É como você é recebido na recepção. É a forma também de como eu trato eles aqui. A forma de como vou ser atendida lá dentro, tudo isso é o acolhimento. Porque se você é bem recebido na recepção você vai ser bem recebido pelo seu médico lá, se você trata eles bem, eles vão te tratar bem lá. Daí então você vai ter o acolhimento dos dois lados ... você vai ter um acolhimento melhor [...] quantas pessoas que trabalham aqui e moram do outro lado da cidade que também tem filho, e que larga com os outros para trabalhar e dar atenção para a gente. Acho que nem todos pensam assim (11).

Figura 2 - Acolhimento uma via de mão dupla, Minas Gerais, Brasil, 2019.

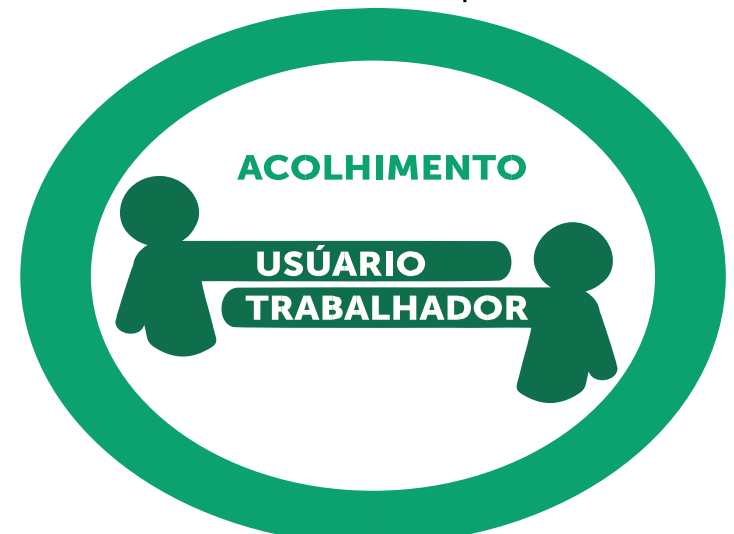

Fonte: Maciel MAS, Silva PCS, Oliveira LF, 2019. 
As evidências científicas apontam, tanto em países desenvolvidos quanto naqueles menos desenvolvidos, que a atenção primária à saúde representa um modelo estratégico e eficaz de resolver as necessidades de saúde da população por meio das redes e do diálogo entre profissionais (CONILL EM et al., 2011).

Já no Brasil, o acolhimento é considerado uma ferramenta que permite colocar em prática os princípios doutrinários e organizativos do SUS, ou seja, o usuário, quando procura o serviço de saúde, procura também resolubilidade, almejando chegar ao serviço e ser acolhido de forma holística (BRASIL, 2013).

Nota-se que os achados do presente estudo corroboram com outras pesquisas sobre 0 acolhimento. Quando o usuário menciona que almeja ser atendido sem pretexto e sem dificuldades, faz uma reflexão do trabalho prestado no sistema de saúde que determina um vínculo palpável de confiabilidade entre os profissionais e os usuários do território de abrangência, tal relação tem como base princípios do Sistema Único de Saúde como, por exemplo, a hierarquização e a regionalização que atendem a demanda da população local á um serviço justo, estendido, integrante e inerente às prerrogativas humanas primordiais. (CARVALHO CA, 2008; BARALDI DC e SOUTO BG, et al., 2011).

Nesse contexto da avaliação do serviço de saúde na atenção primária, quanto a sua qualidade, é importante salientar que o acolhimento enquanto postura refere-se a uma manifestação pró-ativa que fortalece o vínculo do serviço com os usuários na responsabilização do cuidar da saúde individual e comunitária. Desse modo, observa-se que os usuários qualificam o serviço sempre que a sua demanda é respondida (GUERRERO P et al., 2013), o que resulta na efetivação do princípio organizativo do SUS, resolutividade.

\section{0 atendimento quanto à quantidade e o tempo}

O acolhimento ainda é visto pelos usuários como um atendimento que necessita de um número razoável de pessoas, para assim atender à demanda do serviço. Como referido na fala a seguir, o usuário do serviço relata que está faltando pessoal, ou seja, sem um número suficiente o profissional não consegue "despachar" o serviço rapidamente:

"eu acho que está faltando mais só pessoal para trabalhar, às vezes você vem cá fica a tarde toda para ser atendido, então está faltando mais é profissional para atender melhor e despachar logo" (1).

Essa visão centrada no atendimento minimiza o acolhimento a uma estratégia isolada, pois o acolhimento vai muito além dessa visão limitante, entretanto o número de profissionais também é um fator importante para que se alcance a qualidade almejada. Nesse aspecto, há dois lados da moeda; de um lado, o financiamento e a gestão e, do outro, a formação e o trabalho em saúde que devem ser compreendidos como elementos que se complementam e não como polos separados (TEIXEIRA CF, 2003).

O usuário, muitas vezes, está centrado não na qualidade do serviço, mas sim na agilidade com que o serviço é ofertado, observando-se, assim, contradição no movimento de procura da comunidade pelos serviços. Entende-se pela fala a seguir que, se o atendimento foi rápido, o usuário considera que foi bem atendido, quando ele expressa que já passou pelo enfermeiro e pelo médico e já está fazendo o tratamento, o que ele enfatiza pela expressão, "assim eu gosto", ou seja, assim estou satisfeito com o serviço, sem realmente se dar conta do que é o acolhimento. Esse não é um conceito imposto, mas um conceito em construção, focado em um processo produtivo que aposta nas tecnologias relacionais, na relação entre trabalhadores e usuários, a partir de escuta qualificada, de responsabilização, compromissado com a resolutividade e com o trabalho multiprofissional (MASSON N et al., 2015).

Bom, é a terceira vez que eu venho aqui e todas as vezes que eu venho aqui fui bem atendida. Fui atendida no tempo certo tanto que eu cheguei aqui com a pressão alta e já passei pelo enfermeiro, já passei pelo médico e estou fazendo tratamento, entendeu? Então para mim assim eu gosto (16).

$\mathrm{Na}$ fala a seguir, tem-se o princípio organizativo do SUS, a resolutividade, sendo reconhecido como a capacidade do serviço em resolver situações relacionadas ao processo saúde/doença, entretanto se verifica 
que a preocupação central do usuário está relacionada ao tempo que leva para que esse problema seja resolvido, prevalecendo mais uma vez a relação do atendimento com a demora:

[...] Eu tenho um probleminha de saúde com relação a minha coluna, meu ombro tem artrose eu tenho tendinite, às vezes eu não consigo ...eu não tenho estudo...tenho que trabalhar braçal não estou dando conta mais é então é assim... eu tenho um papel ali agendado para fazer uns exames com ortopedia e até hoje não saiu já tem um tempinho [...] Acolhimento para mim é resolver o problema [...] a pessoa está precisando resolver o mais rápido possível que você puder (4).

Cabe destacar a importância de estudos como este que envolve a temática acolhimento, que pode ser visto como um potente analisador das relações entre profissionais, gestores e usuários. Essa fala vai de acordo com questões históricas e institucionais das atividades práticas dos profissionais em saúde, no sentido de atender e ter que resolver os problemas de saúde dos usuários, entendendo-se a resolução em muitos casos como processo de simplificação de aspectos complexos, com repostas prontas e escutas articuladas pelas queixas (FORTUNA CM et al., 2017).

De forma semelhante, em outro estudo que avaliou o grau de satisfação dos usuários em relação ao acolhimento, os usuários demonstram insatisfação frente à demora para serem atendidos. Nesse sentido, é urgente e necessária a orientação da população em relação à rede de serviços de saúde oferecidas e ainda uma melhor forma de organização para o atendimento à demanda espontânea dentro das ESF (LIRA LBS et al., 2018). Fica evidente que o conceito de acolhimento para o usuário ainda é construído de acordo com as suas expectativas históricas e pessoais de que ser bem acolhido é ter o seu problema de saúde resolvido em um curto espaço de tempo por um elevado número de profissionais.

\section{CONCLUSÃO}

Com base nos achados deste estudo, conclui-se que o acolhimento na perspectiva do usuário é visto como um atendimento que é por vezes avaliado em relação à qualidade e outras em relação à quantidade dos serviços prestados na APS, não se reconhecendo o conceito de acolhimento descrito na PNH. E, ainda, pode permear o imaginário do usuário que busca um ambiente de saúde resolutivo que organiza uma rica ação de retroalimentação, ou seja, uma via de mão dupla, em que usuário e trabalhadores participam do processo de saúde. Desse modo, o estudo contribui para melhor compreender a percepção do usuário frente ao acolhimento, reconhecendo as limitações desse entendimento para que futuras pesquisas possam ser desenvolvidas com o intuito de empoderar o usuário como ator social na APS, onde se inicia o processo de acolhimento.

\section{REFERÊNCIAS}

1. BACKES DS, et al. O Sistema Único de Saúde idealizado versus o realizado: contribuições da Enfermagem. Revista Latino-Americana de Enfermagem, 2014; 22(6), 1026-1033.

2. BARALDI DC, SOULTO BGA. A demanda do acolhimento em uma unidade de saúde da família em São Carlos, São Paulo. Arquivos Brasileiros de Ciência da Saúde, 2011; 36(1), 10-17.

3. BRASIL. Ministério da Saúde. Política Nacional de Humanização (PNH): documento base para gestores e trabalhadores do SUS. 4.ed. Brasília: Ministério da Saúde, 2007.

4. BRASIL. Ministério da Saúde. HumanizaSUS: Política Nacional de Humanização. Brasília, DF: Ministério da Saúde; 2013.

5. BRASIL. Ministério da Saúde. Portaria n 2.436, de 21 de setembro de 2017. Aprova a Política Nacional de Atenção Básica, estabelecendo a revisão de diretrizes para a organização da Atenção Básica, no âmbito do Sistema Único de Saúde (SUS). Brasília, DF: Ministério da Saúde, 2017.

6. BRASIL. MINISTÉRIO DA SAÚDE. HumanizaSUS: Acolhimento com avaliação e classificação de risco: um paradigma ético-estético no fazer em saúde. Brasília, 2004. p. 18.

7. CARVALHO CAP, et al. Acolhimento aos usuários: uma revisão sistemática do atendimento no Sistema Único de Saúde. Arquivo de Ciência da Saúde, 2008; 15(2), 93-98. 
8. CONILL EM, et al. Waiting lists in public systems: from expanding supply to tymely access? Reflections on spain's nacional health system. Ciência \& Saúde Coletiva, 2011; 16(6): 2783-2794.

9. FERREIRA BR, et al. Acolhimento ao Idoso na Atenção Básica: Visão do Usuário. Revista de Pesquisa: Cuidado é Fundamental Online, 2018; 10(3):669-674.

10. FORTUNA CM, et al. Embracement as an analyzer of the relationships between professionals, managers and users. Revista da Escola de Enfermagem da USP, 2017; 51:03251 - 03258.

11. GUERRERO $P$, et al. $O$ acolhimento como boa prática na atenção básica à saúde. Texto contexto - enfermagem, $2013 ; 22(1): 132-140$.

12. LIRA LBS, et al. Acesso, acolhimento e estratégia saúde da família: satisfação do usuário, Revista de Enfermagem UFPE on line, 2018; 12(9):2334-2340.

13. LOPES AS, et al. O acolhimento na atenção básica em saúde: relações de reciprocidade entre trabalhadores e usuários. Saúde Debate, 2015;39(104):114-123.

MASSON N, et al. Acolhimento e vínculo: tecnologias relacionais na produção da saúde, Revista Brasileira de Pesquisa em Saúde, 2015; 17(2): 103-110.

14. MINAYO MCS. O desafio do conhecimento: pesquisa qualitativa em saúde. 14ed. São Paulo: Hucitec, $2014 ;$ p. 407.

15. MOROSINI MVGC, et al. Política nacional de atenção básica 2017: retrocessos e riscos para o sistema único de saúde. Saúde em Debate, 2018; 42:11-24.

16. SANTOS DDS, et al. Processo de trabalho na Estratégia de Saúde da Família: potencialidades da subjetividade do cuidado para reconfiguração do modelo de atenção. Ciência \& Saúde Coletiva, 2018; 23: 861-870.

17. TEIXEIRA CF. A mudança do modelo de atenção à saúde no SUS: desatando nós, criando laços. Saúde Debate, 2003; 27(65):257-277. 